\title{
A pathfinder for imaging exo-Earths
}

\author{
SCExAO is an instrument on the Subaru Telescope that is pushing the frontiers of what is possible with ground- \\ based direct imaging of terrestrial exoplanets, explains Thayne Currie, on behalf of the SCExAO team.
}

O ver the past ten years, high-contrast imaging systems and now dedicated extreme adaptive optics $(\mathrm{AO})$ systems on ground-based telescopes have revealed the first direct detections of young, self-luminous Jovian extrasolar planets on Solar System-like scales, up to one million times fainter than the stars they orbit ${ }^{1}$. A key goal of the upcoming 30-m-class Extremely Large Telescopes (ELTs) is to image a rocky, Earth-sized planet in the habitable zone around a nearby $\mathrm{M}$ star. However, doing so requires achieving planetto-star contrasts 100 times deeper at 10 times closer angular separations than currently demonstrated with leading instruments like the Gemini Planet Imager (GPI) or SPHERE at the Very Large Telescope.

The Subaru Coronagraphic Extreme Adaptive Optics project (SCExAO) ${ }^{2}$ utilizes and will further mature key advances in wavefront control and coronagraphy needed to someday allow the imaging of an Earthlike planet with ELTs. The system takes as input and then sharpens a partially corrected wavefront from Subaru's facility AO system (AO188). Subaru's 2,000-actuator deformable mirror is driven by a modulated Pyramid wavefront sensor (WFS) ${ }^{3}$. Owing to these Pyramid optics and an advanced camera used for wavefront sensing $\left(\mathrm{OCAM}^{2} \mathrm{~K}\right)$, SCExAO can target fainter stars than possible with the spatially filtered Shack-Hartmann WFSs used by GPI and SPHERE and its wavefront control loop can run faster (in practice at 2 $\mathrm{kHz}$, and in principle up to $3.5 \mathrm{kHz}$ ). The host star's halo is further suppressed by a suite of versatile coronagraphs.

Starlight is fed into different optical/nearinfrared (IR) science instruments - for exoplanet imaging this is primarily CHARIS, a near-IR integral field spectrograph ${ }^{4}$. CHARIS operates in two modes. Its low-resolution $(R \sim 20)$ 'broadband mode' covering the three major near-IR bandpasses (J, $\mathrm{H}$ and $\mathrm{K}$ ) is well-suited for exoplanet discovery and coarse spectral characterization. A higher-resolution $(R$ $\sim 70$ ) mode in J, $\mathrm{H}$ or $\mathrm{K}$ allows for more detailed, follow-up characterization.

Presently, SCExAO delivers high-quality AO corrections: $\sim 90 \%$ Strehl ratio at $1.6 \mu \mathrm{m}$ under good conditions, and planet-to-star contrasts $\left(\sim 10^{-6}\right.$ at $\left.0.5^{\prime \prime}\right)$ similar to those from GPI and SPHERE and far superior to those achieved with facility AO systems (Fig. 1). It has reached extreme-AO-like contrasts on

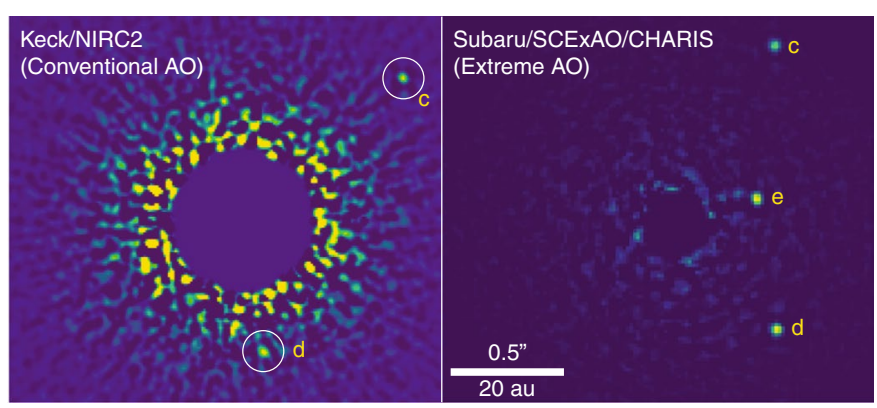

Fig. 1 | Two near-infrared images of the HR 8799 system. The left image is from a leading conventional (facility) AO system using the near-infrared camera on the Keck telescope (NIRC2); on the right is a SCEXAO/CHARIS image, which exhibits higher signal-to-noise detections of planets HR 8799c and HR 8799d and a detection of HR 8799e.

stars as faint as 12 th magnitude in the optical. These deep contrasts translate into a new exoplanet discovery space and higher-fidelity spectra for known directly imaged exoplanets.

The first year of full science operations for SCExAO and CHARIS has revealed insights about massive exoplanets and planet candidates. Most notably, it provided the first high-quality JHK spectrum of Kappa Andromedae b ( $\kappa$ And $b$ ), an object of debatable nature - young exoplanet or intermediate-aged brown dwarf? The SCExAO/CHARIS spectrum for $\kappa$ And $b$ showed evidence for a low surface gravity and similarity to young objects in the planet-mass regime ${ }^{5}$. On the other hand, SCExAO/CHARIS data showed that candidate protoplanets around $\mathrm{LkCa} 15$ and HD 163296, thought to be located within cleared regions of protoplanetary disks, are instead likely misidentified disk signals ${ }^{6,7}$. Even with current capabilities, SCExAO/ CHARIS imaging of many nearby young stars as a part of an upcoming intensive survey should be able to discover Jovian planets on Solar System scales (5-30 au).

Over the next five years, SCExAO will operate both as an exoplanet discovery/ characterization engine and also as a key technology pathfinder for high-contrast imaging with ELTs. A critical advance will be the maturation of new wavefront sensing and control techniques such as predictive control and sensor fusion ${ }^{8}$. Ultra-low-noise detectors ${ }^{9}$ can drive focalplane wavefront sensing/control at high speed and accuracy, yielding a factor of 10-100 gain in planet-to-star contrasts, sufficient to image some exoplanets in reflected light. The general architecture of high-contrast imaging with SCExAO - a two-stage AO system with multiple, complementary loops for focal-plane wavefront sensing - resembles the general architecture envisioned for extreme AO systems on some ELTs (for example, the Planetary Systems Imager on the Thirty Meter Telescope ${ }^{10}$ ).

With SCExAO and other upcoming systems being developed over the next decade as proving grounds (for example, Magellan/MagAO-X, Keck/KPIC and Gemini/GPI-2.0), ELTs may be capable of directly detecting and characterizing the atmospheres of rocky, habitable-zone planets around the nearest $M$ stars within the next 15-20 years.

\section{Thayne Currie}

NASA Ames Research Center, Mountain View, CA, USA.

e-mail: thayne.m.currie@nasa.gov

Published online: 9 May 2019

https://doi.org/10.1038/s41550-019-0773-4

\footnotetext{
References

1. Marois, C. et al. Science 322, 1348-1352 (2008)

2. Jovanovic, N. et al. Publ. Astron. Soc. Pac. 127, 890 (2015).

3. Lozi, J. et al. Publ. Astron. Soc. Pac. 131, 044503 (2019).

4. Groff, T. D. et al. Proc. SPIE 9605, 96051C (2015).

5. Currie, T. et al. Astron. J. 156, 291 (2018).

6. Rich, E. A. et al. Astrophys. J. 875, 38 (2019).

. Currie, T. et al. Astrophys. J. Lett. (in the press).

8. Males, J. R. \& Guyon, O. J. Astron. Telesc. Instrum. Syst. 4, 019001 (2018).

9. Mazin, B. A. et al. Opt. Express 20, 1503 (2012).

10. Guyon, O. et al. Proc. SPIE 10703, 107030Z (2018).
} 